\title{
Relativistic ideal Fermi gas at zero temperature and preferred frame
}

\author{
K. Kowalski, J. Rembieliński and K.A. Smoliński \\ Department of Theoretical Physics, University of Łódź, \\ ul. Pomorska 149/153, 90-236 Łódź, Poland
}

\begin{abstract}
We discuss the limit $T \rightarrow 0$ of the relativistic ideal Fermi gas of luxons (particles moving with the speed of light) and tachyons (hypothetical particles faster than light) based on observations of our recent paper: K. Kowalski, J. Rembieliński and K.A. Smoliński, Phys. Rev. D 76, 045018 (2007). For bradyons this limit is in fact the nonrelativistic one and therefore it is not studied herein.

PACS numbers: 03.30.+p, 05.20.-y, 05.30.-d, 05.70.-a, 05.70.Ce
\end{abstract}


In our very recent paper [1] the Lorentz covariant formulation has been introduced of classical and quantum statistical mechanics and thermodynamics of an ideal gas of relativistic particles. An advantage of this formulation based on the preferred frame approach is among others the possibility of a consistent free of paradoxes description of tachyons. In our discussion of the limit $T \rightarrow 0$ in the case of the relativistic quantum ideal gas we restricted in [1] to the case of the Bose gas. In this work we complete the results given in [1] by considering the limit $T \rightarrow 0$ for the Fermi gas. As with the Bose gas, we do not discuss the limit $T \rightarrow 0$ in the case of bradyons (particles slower than light) because this limit is in fact the nonrelativistic one [2]. Consider first the degenerate Fermi gas of tachyons. The covariant forms of thermodynamic functions for that gas derived in [1] are given by

$$
\begin{aligned}
\frac{U}{V} & =\frac{m^{2}}{2 \pi^{2} u_{0}^{2} \beta^{2}} \sum_{n=1}^{\infty} \frac{(-1)^{n+1}}{n^{2}}\left[3 S_{0,2}\left(n u_{0} \beta m\right)-n u_{0} \beta m S_{-1,1}\left(n u_{0} \beta m\right)\right] s^{n}, \\
\frac{p}{k T} & =\frac{m^{2}}{2 \pi^{2} u_{0}^{2} \beta} \sum_{n=1}^{\infty} \frac{(-1)^{n+1}}{n^{2}} S_{0,2}\left(n u_{0} \beta m\right) s^{n}, \\
\frac{N}{V} & =\frac{m^{2}}{2 \pi^{2} u_{0}^{2} \beta} \sum_{n=1}^{\infty} \frac{(-1)^{n+1}}{n} S_{0,2}\left(n u_{0} \beta m\right) s^{n},
\end{aligned}
$$

where $\beta=1 /(k T), m$ is a mass of a particle, $s=e^{\beta \mu}$ is the fugacity, $\mu$ is the chemical potential, $u_{0}$ is the zeroth (covariant) component of the four velocity $u^{\mu}$ of the preferred frame with repect to the inertial observer (in the preferred frame $u_{0}=1$ ), and $S_{\mu, \nu}(x)$ is the Lommel function (see the appendix). Using (A.5) we obtain the following asymptotic form of (1), (2), and (3) in the limit $T \rightarrow 0$ (i.e. $\beta \rightarrow \infty$ ):

$$
\begin{aligned}
\frac{U}{V} & =\frac{m}{\pi^{2} u_{0}^{3} \beta^{3}} \sum_{n=1}^{\infty} \frac{(-1)^{n+1}}{n^{3}} s^{n}, \\
\frac{p}{k T} & =\frac{m}{2 \pi^{2} u_{0}^{3} \beta^{2}} \sum_{n=1}^{\infty} \frac{(-1)^{n+1}}{n^{3}} s^{n}, \\
\frac{N}{V} & =\frac{m}{2 \pi^{2} u_{0}^{3} \beta^{2}} \sum_{n=1}^{\infty} \frac{(-1)^{n+1}}{n^{2}} s^{n} .
\end{aligned}
$$

Now applying the asymptotic form of the Fermi functions

$$
f_{\nu}(x)=\sum_{n=1}^{\infty} \frac{(-1)^{n+1}}{n^{\nu}} x^{n}
$$


known as Sommerfeld's lemma [3]], such that

$$
\begin{aligned}
& f_{\nu}\left(e^{\beta \mu}\right) \\
& \simeq \frac{(\beta \mu)^{\nu}}{\Gamma(\nu+1)}\left[1+\nu(\nu-1) \frac{\pi^{2}}{6}(\beta \mu)^{-2}+\nu(\nu-1)(\nu-2)(\nu-3) \frac{7 \pi^{4}}{360}(\beta \mu)^{-4}+\ldots\right], \quad \beta \mu \gg 1
\end{aligned}
$$

we find

$$
\begin{aligned}
\frac{U}{V} & =\frac{m \mu^{3}}{6 \pi^{2} u_{0}^{3}}\left(1+\pi^{2} \frac{k^{2} T^{2}}{\mu^{2}}\right), \\
p & =\frac{m \mu^{3}}{12 \pi^{2} u_{0}^{3}}\left(1+\pi^{2} \frac{k^{2} T^{2}}{\mu^{2}}\right), \\
\frac{N}{V} & =\frac{m \mu^{2}}{4 \pi^{2} u_{0}^{3}}\left(1+\frac{\pi^{2}}{3} \frac{k^{2} T^{2}}{\mu^{2}}\right) .
\end{aligned}
$$

We remark that as with the case of the Bose gas of tachyons [1], (9) and (10) imply the equation of state of the form

$$
p V=\frac{U}{2}
$$

Now, it follows immediately from (11) that the Fermi energy $\varepsilon_{\mathrm{F}}$ is given by

$$
\varepsilon_{\mathrm{F}}=\sqrt{\frac{4 \pi^{2} u_{0}^{3} N}{m V}},
$$

where $\varepsilon_{\mathrm{F}}=\mu(T=0)$. Furthermore, eqs. (11) and (13) yield

$$
\varepsilon_{\mathrm{F}}^{2}=\mu^{2}+\frac{\pi^{2}}{3} k^{2} T^{2}
$$

which leads to

$$
\mu=\varepsilon_{\mathrm{F}}-\frac{\pi^{2}}{6 \varepsilon_{\mathrm{F}}} k^{2} T^{2}
$$

We point out that in this section we perform calculations up to terms of order $T^{2}$. Making use of (14) and (15) we can write (9) and (10) in the following form:

$$
\begin{aligned}
U & =\frac{m V}{6 \pi^{2} u_{0}^{3}}\left(\varepsilon_{\mathrm{F}}^{3}+\frac{\pi^{2}}{2} \varepsilon_{\mathrm{F}} k^{2} T^{2}\right), \\
p & =\frac{m}{12 \pi^{2} u_{0}^{3}}\left(\varepsilon_{\mathrm{F}}^{3}+\frac{\pi^{2}}{2} \varepsilon_{\mathrm{F}} k^{2} T^{2}\right) .
\end{aligned}
$$

Consider now the entropy which can be defined as

$$
S=\frac{U+p V-\mu N}{T} .
$$

Taking into account (9), (10), (11) and (15) we get

$$
S=\frac{m k^{2}}{6 u_{0}^{3}} V \varepsilon_{\mathrm{F}} T .
$$


Therefore, the entropy vanishes at $T=0$. The formula (19) can be also obtained from the well-known relation

$$
S=\int_{0}^{T} \frac{C_{V}}{T} d T,
$$

where

$$
C_{V}=\left(\frac{\partial U}{\partial T}\right)_{V}=\frac{m k^{2}}{6 u_{0}^{3}} V \varepsilon_{\mathrm{F}} T,
$$

which is immediate consequence of (16).

Finally, we study the limit $T \rightarrow 0$ in the case of the Fermi gas of luxons. Using the formulas on thermodynamic functions derived in [1] such that

$$
\begin{aligned}
\frac{U}{V} & =\frac{3}{\pi^{2} u_{0}^{4} \beta^{4}} \sum_{n=1}^{\infty} \frac{(-1)^{n+1}}{n^{4}} s^{n}, \\
\frac{p}{k T} & =\frac{1}{\pi^{2} u_{0}^{4} \beta^{3}} \sum_{n=1}^{\infty} \frac{(-1)^{n+1}}{n^{4}} s^{n}, \\
\frac{N}{V} & =\frac{1}{\pi^{2} u_{0}^{4} \beta^{3}} \sum_{n=1}^{\infty} \frac{(-1)^{n+1}}{n^{3}} s^{n},
\end{aligned}
$$

and proceeding analogously as with tachyons, we obtain

$$
\begin{aligned}
\frac{U}{V} & =\frac{\mu^{4}}{8 \pi^{2} u_{0}^{4}}\left(1+2 \pi^{2} \frac{k^{2} T^{2}}{\mu^{2}}\right), \\
p & =\frac{\mu^{4}}{24 \pi^{2} u_{0}^{4}}\left(1+2 \pi^{2} \frac{k^{2} T^{2}}{\mu^{2}}\right), \\
\frac{N}{V} & =\frac{\mu^{3}}{6 \pi^{2} u_{0}^{4}}\left(1+\pi^{2} \frac{k^{2} T^{2}}{\mu^{2}}\right) .
\end{aligned}
$$

We point out that (25) and (26) imply the well-known equation of state for ideal gas of massless particless such that

$$
p V=\frac{U}{3} .
$$

Furthermore, the Fermi energy is

$$
\varepsilon_{\mathrm{F}}=\left(6 \pi^{2} u_{0}^{4} \frac{N}{V}\right)^{\frac{1}{3}} .
$$

Hence, using (27) we find

$$
\varepsilon_{\mathrm{F}}^{3}=\mu^{3}+\pi^{2} k^{2} T^{2} \mu,
$$

implying

$$
\mu=\varepsilon_{\mathrm{F}}-\frac{\pi^{2}}{3 \varepsilon_{\mathrm{F}}} k^{2} T^{2} .
$$


The relations (25) and (26) written in terms of the Fermi energy take the form

$$
\begin{aligned}
U & =\frac{V}{8 \pi^{2} u_{0}^{4}}\left(\varepsilon_{\mathrm{F}}^{4}+\frac{2 \pi^{2}}{3} \varepsilon_{\mathrm{F}}^{2} k^{2} T^{2}\right), \\
p & =\frac{1}{24 \pi^{2} u_{0}^{4}}\left(\varepsilon_{\mathrm{F}}^{4}+\frac{2 \pi^{2}}{3} \varepsilon_{\mathrm{F}}^{2} k^{2} T^{2}\right) .
\end{aligned}
$$

The entropy calculated with the help of (18) is

$$
S=\frac{k^{2}}{6 u_{0}^{4}} V \varepsilon_{\mathrm{F}}^{2} T
$$

Thus, as with tachyons, the entropy in the case of fermionic luxons also vanishes. The above formula on the entropy is also implied by (20) and

$$
C_{V}=\left(\frac{\partial U}{\partial T}\right)_{V}=\frac{k^{2}}{6 u_{0}^{4}} V \varepsilon_{\mathrm{F}}^{2} T
$$

following directly from (32).

In conclusion, we have derived in this work the Lorentz covariant form of thermodynamic functions of Fermi ideal gas of luxons and tachyons in the zero temperature limit. It seems that the observations of this paper would be of interest for testing the hypothesis of tachyonic neutrinos [4] and tachyonic dark matter [5]. Indeed, the existing estimations of density of neutrinos suggest that the model of an ideal gas is appropriate for the neutrino background.

\section{Acknowledgements}

This paper has been supported by University of Lodz grant.

\section{APPENDIX}

We recall some basic properties of the Lommel functions $S_{\mu, \nu}(x)[6]$. The Lommel functions $S_{0, \nu}(x)$ have the integral representation such that

$$
S_{0, \nu}(x)=\int_{0}^{\infty} e^{-x \sinh t} \cosh \nu t d t=\frac{x}{\nu} \int_{0}^{\infty} e^{-x \sinh t} \cosh t \sinh \nu t d t, \quad x>0 .
$$

The Lommel functions $S_{\nu, \nu}(x)$ can be expressed by means of the Struve functions $\mathbf{H}_{\nu}(x)$ and the Bessel functions $Y_{\nu}(x)$ (Neumann functions) also designated by $N_{\nu}(x)$. Namely, we have

$$
S_{\nu, \nu}(x)=2^{\nu-1} \sqrt{\pi} \Gamma\left(\nu+\frac{1}{2}\right)\left[\mathbf{H}_{\nu}(x)-Y_{\nu}(x)\right]
$$


The Lommel functions $S_{\mu, \nu}(x)$ are defined recurrently

$$
\begin{gathered}
S_{\mu+2, \nu}(x)=x^{\mu+1}-\left[(\mu+1)^{2}-\nu^{2}\right] S_{\mu, \nu}(x), \\
S_{\mu, \nu}^{\prime}(x)=\frac{\nu}{x} S_{\mu, \nu}(x)+(\mu-\nu-1) S_{\mu-1, \nu+1}(x)=-\frac{\nu}{x} S_{\mu, \nu}(x)+(\mu+\nu-1) S_{\mu-1, \nu-1}(x) .
\end{gathered}
$$

The asymptotics $S_{0,2}(x)$ for $x \gg 1$ is of the form

$$
S_{0,2}(x)=\frac{1}{x}, \quad x \gg 1
$$

[1] K. Kowalski, J. Rembieliński and K.A. Smoliński, Phys. Rev. D 76, 045018 (2007).

[2] C. Aragão de Carvalho and S. Goulart Rosa Jr, J. Phys. A 13, 3233 (1980).

[3] R.K. Pathria, Statistical Mechanics (Pergamon, New York, 1977).

[4] J. Ciborowski and J. Rembieliński, Eur. Phys. J. C 8, 157 (1999); A. Chodos, A.I. Hauser and A.V. Kostelecký, Phys. Lett. B 150, 431 (1985).

[5] P.C.W. Davies, Internat. J. Theoret. Phys. 43, 141 (2004); A. Das et al, Phys. Rev. D 72, 043528 (2005).

[6] I.S. Gradshteyn and I.M. Ryzhik, Tables of Integrals, Series, and Products (Academic Press, New York, 2000); H. Bateman, Higher Transcendental Functions, vol. 2, (McGraw-Hill, New York, 1953). 